\title{
High Precision Timing of PSR J0437-4715
}

\author{
M. C. Britton, W. van Straten, M. Bailes, M. Toscano \\ Swinburne Centre for Astrophysics and Supercomputing, Swinburne \\ University of Technology, P.O. Box 218, Hawthorn, Victoria 3122, \\ Australia \\ R. N. Manchester \\ Australia Telescope National Facility, CSIRO, Epping NSW 2121, \\ Australia
}

\begin{abstract}
Systematic effects currently dominate the timing residuals of the pulsar PSR J0437-4715. These systematics arise from transformations that occur during the propagation and detection of radio waves. The transformation properties of polarized radiation are related to the Lorentz group, and such effects may be represented using Lorentz transformations. These effects may be removed using the techniques of polarimetric selfcalibration. Alternatively, an invariant profile may be formed from the polarimetric analogue of the Lorentz invariant $I^{2}-Q^{2}-U^{2}-V^{2}$ and used for pulsar timing. Observations of PSR J0437-4715 are presented that show the consistency between these two techniques.
\end{abstract}

\section{Introduction}

Systematic effects currently dominate the timing residuals of the pulsar PSR J0437-4715 (Sandhu et al. 1997). The timing accuracy for this pulsar is of order $500 \mathrm{~ns}$, whereas arguments based on its pulse width and signal-to-noise ratio suggest that the accuracy should be an order of magnitude better than this. For a timing parameter whose precision scales as the square root of the integration time, this constitutes a factor of 100 in telescope time. These systematics are caused by variations in the shape of the pulse profile, which may arise from transformations that occur during the propagation and detection of radio waves. Effects such as Faraday rotation, receiver cross-talk and differential amplification act to mix the four Stokes parameters, thereby changing the shape of the polarimetric pulse profiles. These effects may vary in time and frequency, causing the pulse profiles to vary as well. Such variations have long been known to corrupt polarimetric observations (Stinebring et al. 1984). They may also be the dominant source of systematic error for high precision timing observations as well, because small changes in the shape of the total intensity pulse profile shift the measured time of arrival (TOA). Thus, at some level of precision, timing of the total intensity becomes inextricably linked to polarimetry. 


\section{Radio-Astronomical Polarimetry}

To investigate the transformations that arise during the propagation and detection of radio waves, it is useful to have a context with which to describe them. The linear transformations of polarized radiation are described by the Lorentz group (Brown \& Bak 1995; Britton 2000a). Briefly, polarized radiation may be represented by the analytic signal $\vec{E}$. The analytic signal transforms under multiplication by $2 \times 2$ complex matrices called Jones matrices. The set of such transformations constitutes the group $\mathrm{SL}(2, \mathrm{C})$, which forms the spin $1 / 2$ representation of the Lorentz group. Alternatively, polarized radiation may be described by the Stokes parameters $S=(I, Q, U, V)$, which transform under multiplication by $4 \times 4$ real matrices called Mueller matrices. The set of Mueller matrices corresponding to transformations of the analytic signal form the group $\mathrm{SO}(3,1)$, which forms the spin 1 representation of the Lorentz group. That is, the Stokes parameters transform as a Lorentz 4 -vector, with the total intensity acting as the timelike component and the remaining 3 Stokes parameters acting as the spacelike components.

Using this formalism, the effects that arise during the propagation and detection of radio waves may be represented in terms of a series of Lorentz transformations acting on the Stokes parameters $S$ to produce the observed Stokes parameters $S^{\prime}$.

$$
\begin{aligned}
S^{\prime}= & g_{a} g_{b} \mathbf{B}_{\hat{q}}(2 \beta) \mathbf{R}_{\hat{q}}\left(2 \Phi_{I}\right) \mathbf{B}_{\hat{u}}^{(1)}\left(\delta_{\theta}\right) \mathbf{B}_{\hat{v}}^{(1)}\left(\delta_{\chi}\right) \mathbf{R}_{\hat{u}}^{(1)}\left(\sigma_{\chi}\right) \mathbf{R}_{\hat{v}}\left(\sigma_{\theta}\right) \\
& \mathbf{R}_{\hat{v}}(2 \zeta) \mathbf{R}_{\hat{v}}\left(2 \Phi_{\text {iono }}\right) \mathbf{R}_{\hat{v}}\left(2 \Phi_{\mathrm{ISM}}\right) S
\end{aligned}
$$

Here the transformations $\mathbf{R}_{\hat{n}}$ and $\mathbf{B}_{\hat{n}}$ denote rotations and boosts with respect to the $\hat{n}$ axis in the space of the Poincare sphere. The angles $\Phi_{\text {iono }}$ and $\Phi_{\text {ISM }}$ arise from Faraday rotation in the ionosphere and interstellar medium, respectively. The angle between the frame of the telescope and that of the sky is represented by $\zeta$. A receiver can be viewed as consisting of two receptors, each sensitive to an arbitrary sense of elliptical polarization. The parameters $\sigma_{\theta}, \sigma_{\chi}$, $\delta_{\theta}$ and $\delta_{\chi}$ represent sums and differences of the axial ratios $\chi$ and orientations $\theta$ of these two ellipses. The superscripts on three of these terms indicate that they are expanded to first order about a linear receptor configuration. The angle $\Phi_{I}$ represents a phase delay between the two detected voltage signals that may arise from differing electronics pathlengths. Finally, $g_{a}$ and $g_{b}$ represent the amplification applied to the voltage signals during reception and in the electronics downconversion chain, while the parameter $\beta=\ln \left(g_{b} / g_{a}\right)$ represents the relative gain.

The formulation of these effects in terms of Lorentz transformations permits a simple interpretation of their consequences. Rotations may change the polarimetric properties of the radiation, but preserve the degree of polarization $|\vec{S}| / S_{o}$, and the total intensity $S_{0}$. Boosts mix the total intensity $S_{0}$ with the polarized component $\vec{S}$. Because the total intensity may be much larger than the polarized component, these transformations can completely corrupt the polarized flux. For high precision timing observations, the mixing of $\vec{S}$ into $S_{0}$ may corrupt the total intensity profile. For example, a $1 \%$ error in $\beta, \delta_{\theta}$ or $\delta_{\chi}$ introduces an error of order $100 \mathrm{~ns}$ in the TOA. 


\section{Polarimetric Calibration and Timing of PSR J0437-4715}

Calibration of polarimetric observations constitutes an inversion of equation 1 . Naturally, such an inversion requires a measurement of all four Stokes parameters as well as a knowledge of the values parameterizing the transformations. Several researchers have used polarimetric self-calibration techniques to facilitate the inversion of this equation, either through observations of radio galaxies with known polarization properties (Turlo, Forkert \& Wilson 1985), or through pulsar observations (Stinebring et al. 1984; Xilouris 1991; Britton 2000b). Pulsar self-calibration relies on the fact that the polarimetric pulse profile emitted by the pulsar is very stable when integrated over several hundred pulses. Thus, any time or frequency dependence in the measured Stokes parameters must arise from the transformations in equation 1 . And since this equation may be applied at each point in pulse phase, the observed Stokes parameters $S^{\prime}$ vastly overdetermine the parameters of the model. With an observation consisting of many independent measurements of the pulse profile, one may perform a nonlinear $\chi^{2}$ fit for the values of the propagation and instrumental effects as well as the Stokes parameters $S$ emitted by the pulsar. For pulsar timing an alternative to this procedure is provided by the existence of a polarimetric analogue of the Lorentz invariant. Up to the multiplicative constant $g_{a} g_{b}$, the quantity $I^{2}-Q^{2}-U^{2}-V^{2}$ is invariant under the transformations in equation 1 . Thus one may form an invariant profile and time this quantity instead of the total intensity without the need for calibration.

To test these techniques, eight hour observations of PSR J0437-4715 were taken for 3 successive days in July, 1997 at the Parkes radiotelescope using the center beam of the multi-beam receiver. The two senses of linear polarization were two-bit sampled and recorded with the $16 \mathrm{MHz}$ S2 baseband recorder centered at $1420.4 \mathrm{MHz}$. Data were coherently dedispersed in software and the Stokes parameters formed (van Straten et al. these proceedings). The data were then folded at the pulse period and integrated for 4 minutes. Each eight-hour set of observations were then fitted to the model of equation 1 . The $5 \%$ of the pulse profile surrounding the peak was used in the fit. Because of the relatively narrow bandwidth of this system, all parameters in equation 1 were assumed constant in frequency. Values of the ionospheric RM, relative gain, and instrumental phase were allowed to vary as a function of time. Values of the Stokes parameters $S$ and the receiver parameters were assumed to be constant in time, and their values were determined by the fit. The results of this fit were used to invert equation 1 and obtain the corrected Stokes parameters. The TOAs were then computed using the total intensity pulse profile. A set of TOAs were also computed by forming the invariant profile from the uncalibrated data. The timing residuals from this analysis are shown in figure 1, which demonstrates the consistency between these techniques.

\section{References}

Britton, M. C. 2000a, ApJ, in press

Britton, M. C., van Straten, W., Bailes, M., Toscano, M. \& Manchester, R. N. $2000 \mathrm{~b}, \mathrm{ApJ}$, in preparation 


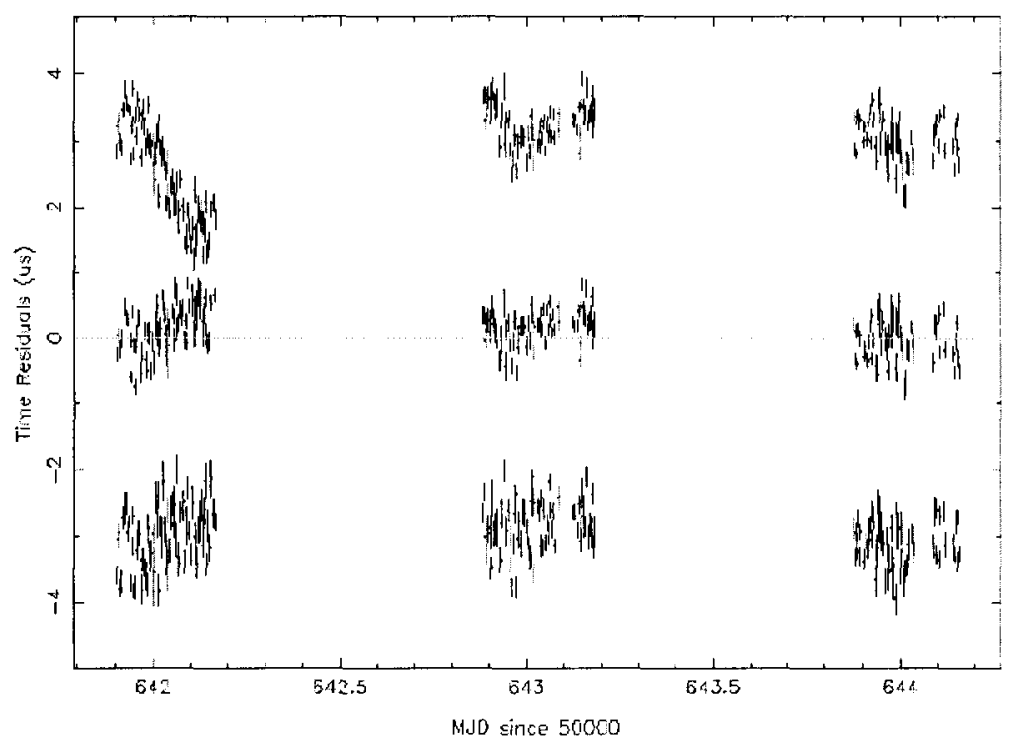

Figure 1. Timing residuals for 3 eight hour observations of PSR J0437-4715 recorded with the $16 \mathrm{MHz}$ S2 baseband system at 1420.4 $\mathrm{MHz}$. Data were reduced in three different ways, and a time offset was introduced between each set of residuals for display purposes. The upper set results from timing uncalibrated data. The middle set was formed from data that had been reduced using polarimetric selfcalibration. The lower set results from forming the invariant profile from uncalibrated data and timing the resulting profile. The consistency between the last two techniques demonstrates their validity.

Brown, C. S. \& Bak, A. E. 1995, Optical Engineering, 34, 1625

Sandhu, J. S., Bailes, M., Manchester, R. N., Navarro, J., Kulkarni, S. R. \& Anderson, S. B. 1997, ApJ, 478, L95

Stinebring, D. R., Cordes, J. M., Rankin, J. M., Weisberg, J. M. \& Boriakoff, V. 1984, ApJS, 55, 247

Turlo, Z., Forkert, T., Sieber, W. \& Wilson, W 1985, A\&A, 142, 181

Xilouris, K. M. 1991, å, 248, 323 